International Journal of Applied Mathematical Research, 1 (4) (2012) 659-665

(C) Science Publishing Corporation

www.sciencepubco.com/index.php/IJAMR

\title{
Using He's Variational Method to Seek the Traveling Wave Solution of PHI-Four Equation
}

\author{
Maliheh Najafi \\ Department of Physiology, Faculty of Medicine, Kermanshah University of \\ Medical Sciences, Kermanshah, Iran \\ malihe_math87@yahoo.com
}

\begin{abstract}
In this paper, we established travelling wave solutions of the nonlinear equation. The He's variational method was used to construct travelling wave solutions of the PHI-four equation. The obtained results include periodic and solitary wave solutions. It is also shown that the He's variational method, with the help of symbolic computation, provides a powerful mathematical tool for solving other nonlinear evolution equations arising in mathematical physics.
\end{abstract}

Keywords: Variational method, Solitary solution, PHI-four equation, periodic solution.

\section{Introduction}

Many phenomena in physics and other fields such as biology, chemistry, mechanics, etc., are described by nonlinear partial differential equations (NLPDEs). As mathematical models of the phenomena, the investigation of exact solutions of NLPDEs will help one to understand these phenomena better. The study of exact solutions of nonlinear evolution equations plays an important role in soliton theory and explicit formulas of nonlinear partial differential equations play an essential role in the nonlinear science. Also, the explicit formulas may provide physical information and help us to understand the mechanism of related physical models.

Recently, many effective methods to construct traveling wave solutions of NLPDEs have been established, such as Adomian decomposition method [1], the 
homotopy perturbation method [2], the variational iteration method $[3,4]$, the He's variational approach [5], the $F$-expansion method [6], three-wave method [7], extended homoclinic test approach $[8,9]$, the $\left(\frac{G^{\prime}}{G}\right)$-expansion method [6] and the exp-function method [10].

In this paper, by means of the He's variational approach, we will obtain some Solitary and periodic solutions of the following PHI-four equation given in [11]

$$
u_{t t}-\alpha u_{x x}-u+u^{3}=0
$$

where $\alpha$ is arbitrary constant.

\section{He's Variational Method}

In order to seek its travelling wave solution, we introduce a transformation

$$
u(x, t)=u(\xi) \quad, \quad \xi=x-c t
$$

by substituting Eqs. (2) into Eq. (1), we have

$$
\left(c^{2}-\alpha\right) u^{\prime \prime}-u+u^{3}=0,
$$

where prime denotes the differential with respect to $\xi$.

According to Ref. [12], By He's semi-inverse method [13], we can arrive at the following variational formulation:

$$
J(\phi)=\int_{0}^{\infty}\left[-\frac{1}{2}\left(c^{2}-\alpha\right)\left(u^{\prime}\right)^{2}-\frac{1}{2} u^{2}+\frac{1}{4} u^{4}\right] d \xi
$$

We assume the soliton solution in the following form

$$
v(\xi)=A \operatorname{sech}(\xi),
$$

where $A$ is an unknown constant to be further determined.

By Substituting Eq. (5) into Eq. (4) we obtain

$$
J=\frac{1}{6} A^{4}-\frac{1}{2} A^{2}-\frac{1}{6} c^{2} A^{2}+\frac{1}{6} A^{2} \alpha,
$$

For making $J$ stationary with respect to $A$

$$
\frac{\partial J}{\partial A}=\frac{2}{3} A^{3}-A-\frac{1}{3} c^{2} A+\frac{1}{3} A \alpha,
$$

From Eq. (7), we have

$$
A= \pm \frac{\sqrt{6+2\left(c^{2}-\alpha\right)}}{2}
$$


The solitary solutions are, therefore, obtained as follows:

$$
u_{11}(\xi)=\frac{\sqrt{6+2\left(c^{2}-\alpha\right)}}{2} \operatorname{sech}(x-c t)
$$

and

$$
u_{12}(\xi)=-\frac{\sqrt{6+2\left(c^{2}-\alpha\right)}}{2} \operatorname{sech}(x-c t)
$$

where $c$ and $\alpha$ are arbitrary constants.

Fig. 1 and Fig. 2 show the graph of $u_{11}(x, t)$ and $u_{12}(x, t)$ for $c=\alpha=1$, $-30 \leq x \leq 30$ and $0 \leq t \leq 10$.

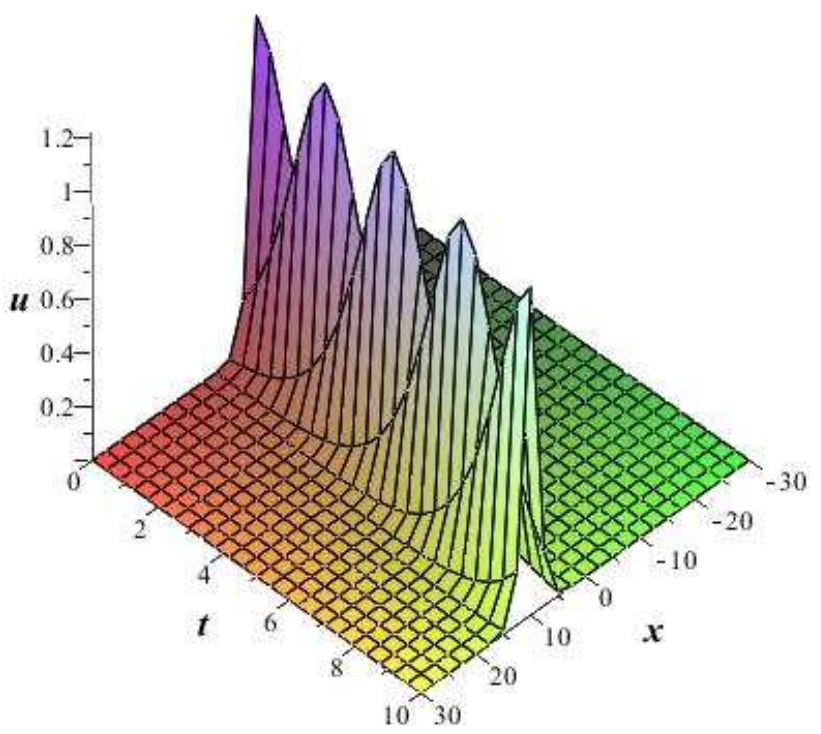

Figure 1: The Soliton solution of Eq. (9).

We search another soliton solution in the form

$$
u(\xi)=D \operatorname{sech}^{2}(\xi)
$$

where $D$ is an unknown constant to be further determined.

By Substituting Eq. (11) into Eq. (4) we obtain

$$
J=-\frac{1}{3} D^{2}+\frac{4}{35} D^{4}-\frac{4}{15} c^{2} D^{2}+\frac{4}{15} D^{2} \alpha,
$$

For making $J$ stationary with respect to $D$

$$
\frac{\partial J}{\partial D}=-\frac{2}{3} D+\frac{16}{35} D^{3}-\frac{8}{15} c^{2} D+\frac{8}{15} D \alpha,
$$




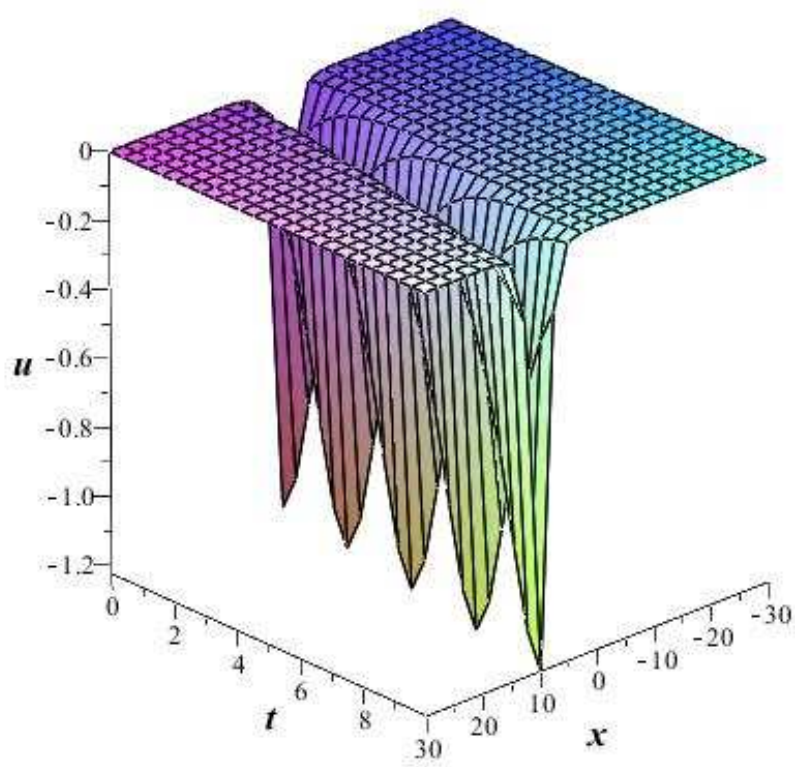

Figure 2: The Soliton solution of Eq. (10).

From Eq. (13), we have

$$
D= \pm \frac{\sqrt{210+168 c^{2}-168 \alpha}}{12}
$$

The solitary solutions are, therefore, obtained as follows:

$$
u_{21}(\xi)=\frac{\sqrt{210+168 c^{2}-168 \alpha}}{12} \operatorname{sech}^{2}(x-c t)
$$

and

$$
u_{22}(\xi)=-\frac{\sqrt{210+168 c^{2}-168 \alpha}}{12} \operatorname{sech}^{2}(x-c t),
$$

where $c$ and $\alpha$ are arbitrary constants.

Fig. 3 and Fig. 4 show the graph of $u_{21}(x, t)$ and $u_{22}(x, t)$ for $c=\alpha=1$, $-30 \leq x \leq 30$ and $0 \leq t \leq 10$. 


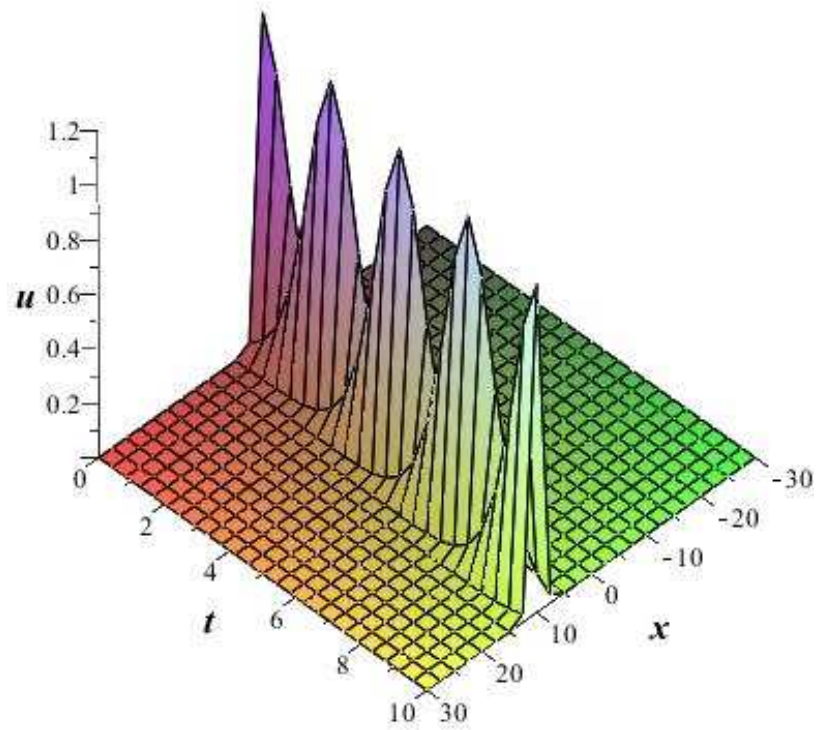

Figure 3: The Soliton solution of Eq. (15).

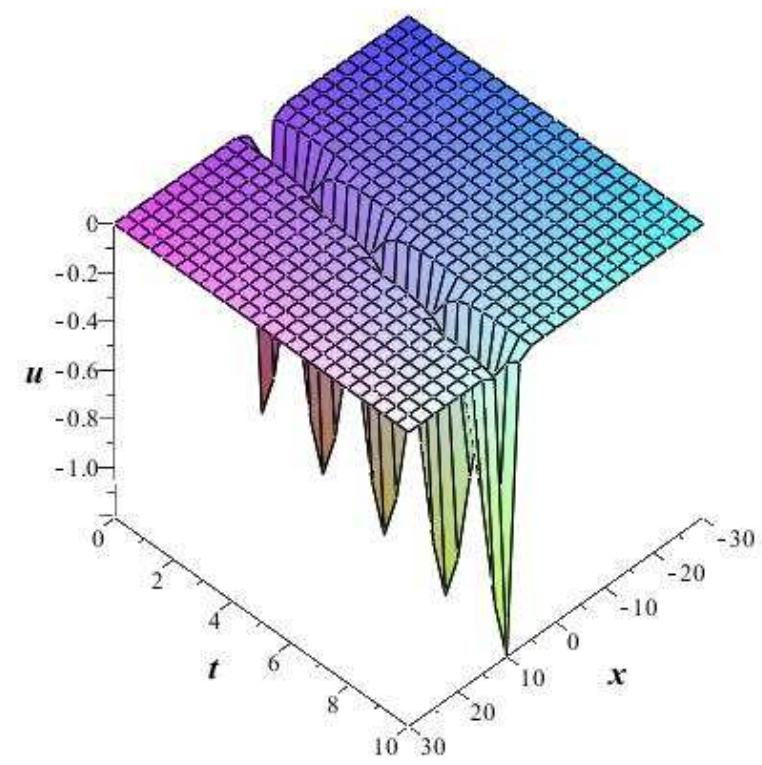

Figure 4: The Soliton solution of Eq. (16).

\section{Conclusion}

In this paper, we used He's variational method to search for solitary solutions of PHI-four equation. This paper shows that the He's variational principle 
provides a very effective and powerful tool for solving nonlinear equations in mathematical physics.

\section{References}

[1] S. Abbasbandy, M.T. Darvishi, A numerical solution of Burgers equation by modified Adomian method, Appl. Math. Comput. 163 (2005) 12651272 .

[2] J.H. He, Application of homotopy perturbation method to nonlinear wave equations, Chaos, Solitons and Fractals 26(3) (2005) 695-700.

[3] J.H. He, X.H. Wu, Construction of solitary solution and compacton-like solution by variational iteration method, Chaos, Solitons and Fractals, 29 (2006) 108-113.

[4] M.T. Darvishi, F. Khani, S. Hamedi-Nezhad, S.-W. Ryu, New modification of the HPM for numerical solutions of the sine-Gordon and coupled sine-Gordon equations, Int. J. Comput. Math. 87(4) (2010) 908-919.

[5] H. Najafi, M. Najafi, S. Arbabi Mohammad-Abadi, New soliton solutions for Kaup-Boussinesq system, International Journal of Applied Mathematical Research, 1 (2012) 402-408.

[6] M.T. Darvishi, Maliheh Najafi, Mohammad Najafi, Traveling wave solutions for the $(3+1)$-dimensional breaking soliton equation by $\left(\mathrm{G}^{\prime} / \mathrm{G}\right)$ expansion method and modified F-expansion method, International Journal of Computational and Mathematical Sciences, 6 (2012) 64-69 .

[7] M.T. Darvishi, M. Najafi, M. Najafi, New exact solutions for the $(3+1)$ dimensional breaking soliton equation, International Journal of Engineering and Mathematical Sciences, 6(2) (2010) 137-140.

[8] M.T. Darvishi, Mohammad Najafi, A modification of extended homoclinic test approach to solve the $(3+1)$-dimensional potential-YTSF equation, Chin. Phys. Lett., 28(4) (2011) 040202.

[9] Mohammad Najafi, Maliheh Najafi, M.T. Darvishi, New exact solutions to the $(2+1)$-dimensional Ablowitz-Kaup-Newell-Segur equation: Modication of extended homoclinic test approach, Chin. Phys. Lett., 29(4) (2012) 040202. 
[10] J.H. He, M.A. Abdou, New periodic solutions for nonlinear evolution equations using Exp-function method, Chaos, Solitons and Fractals 34 (2007) 1421-1429.

[11] X. Denga, M. Zhao, X. Li, Travelling wave solutions for a nonlinear variant of the PHI-four equation, Mathematical and Computer Modelling 49 (2009) 617-622.

[12] J.H. He, Some asymptotic methods for strongly nonlinear equations, Internat. J. Modern Phys. B 20 (10) (2006) 1141-1199.

[13] J.H. He, Variational principles for some nonlinear partial differential equations with variable coefficients, Chaos Solitons Fractals 19 (4) (2004) 847851. 